\title{
PREVALENCIA Y CREENCIAS ASOCIADAS AL CONSUMO DE ALCOHOL EN MUJERES UNIVERSITARIAS
}

\section{PREVALENCE AND BELIEFS ASSOCIATED WITH ALCOHOL CONSUMPTION IN UNIVERSITY WOMEN}

Pedro García García, América Gisel Trejo Lerma, Laura Hinojosa García, Aída Aleyda Jiménez Martínez y Gloria Nelly Gracia Castillo

Universidad Autónoma de Tamaulipas. México

\section{Abstract}

Alcohol is a psychoactive substance with properties that cause physical and mental dependence; Its consumption has been related to metabolic, physiological and behavioral changes. Objective. Measure the prevalence of alcohol consumption, the beliefs of alcohol consumption and the relationship between both variables in university women. Method. Descriptive, cross-sectional and correlational study. The population consisted of 138 university women from a private university in the City of H. Matamoros Tamaulipas, Mexico. A data card and alcohol consumption were applied to measure beliefs, using the decisional balance scale for alcohol consumption prepared by Maddock (1997). Results. A prevalence of alcohol consumption at some time in life of $92.8 \%$ was found. Conclusions. The prevalence of alcohol consumption showed a positive and significant relationship with the pros index for alcohol consumption.

Keywords: Alcohol consumption, beliefs, university women.

\section{Resumen}

El alcohol es una sustancia psicoactiva con propiedades que originan dependencia física y psíquica; su consumo ha sido relacionado con alteraciones metabólicas, fisiológicas y de conducta. Objetivo. Medir la prevalencia de consumo de alcohol, las creencias del consumo de alcohol y la relación entre ambas variables en mujeres universitarias. Método. Estudio descriptivo, transversal y correlacional. La población estuvo constituida por 138 mujeres universitarias de una universidad privada de la Cd. de H. Matamoros Tamaulipas, México. Se aplicó una cedula de datos y consumo de alcohol, para medir las creencias, se utilizó la escala de balance decisional para el consumo del alcohol elaborada por Maddock (1997). Resultados. Se encontró una prevalencia de consumo de alcohol alguna vez en la vida de $92.8 \%$. Conclusiones. Las prevalencias de consumo de alcohol mostraron relación positiva y significativa con el índice de pros para el consumo de alcohol.

Palabras clave: Consumo de alcohol, creencias, mujeres universitarias. 
Para la Organización Mundial de Salud (OMS, 2014), el alcohol es una sustancia psicoactiva con propiedades que originan dependencia física y psíquica; su consumo ha sido relacionado con alteraciones metabólicas, fisiológicas y de conducta debido a la acción depresora que tiene sobre el sistema nervioso central y que, a la vez, ocasiona repercusiones de tipo personal, social y económico. Más recientemente la OMS (2018), reporta que cada año se originan 3.3 millones de muertes en el mundo debido al consumo nocivo de alcohol, lo que constituye un $5.9 \%$ de todas las defunciones, además de ser un factor causal en más de 200 enfermedades y trastornos a la salud. La Organización Panamericana de la Salud (OPS, 2015), de acuerdo al "Informe de situación regional sobre el alcohol y la salud en las Américas", reporta que, por término medio, en las Américas se consume más alcohol que en el resto del mundo. En particular, en los últimos cinco años han aumentado los episodios de consumo excesivo, pasando de una tasa del $4.6 \%$ al $13 \%$ en el caso de las mujeres y del $17.9 \%$ al $29.4 \%$ en el de los hombres.

En México, según el reporte de la Encuesta Nacional de Consumo de Drogas Alcohol y Tabaco 2016-2017 (ENCODAT, 2017) documenta que el 71\% de la población entre 12 y 65 años refirió haber consumido alcohol alguna vez en la vida ( $80.1 \%$ hombres y $62.6 \%$ mujeres); 49.1\% (41.8 millones) en el último año (59.8\% hombres y $39 \%$ mujeres) y el $35.9 \%$ en el último mes (48.1\% hombres y $24.4 \%$ mujeres). El consumo excesivo de alcohol en el último mes se presentó en el 19.8\% (16.8 millones) de la población; $29.9 \%$ hombres y $10.3 \%$ mujeres. En Tamaulipas el $44.2 \%$ de la población estudiantil ha consumido alcohol alguna vez en la vida (44.8\% hombres y $43.7 \%$ mujeres) y el $10.5 \%$ ha presentado consumo excesivo de alcohol (11\% hombres y $9.9 \%$ mujeres). Específicamente en la ciudad de $\mathrm{H}$. Matamoros Tamaulipas, García et al., (2016) en un estudio con adolescentes encontraron que el $65.6 \%$ de estos habían consumido alcohol alguna vez en la vida; un 41.8\% (135) continuó con el consumo en el último año, el 16.4\% (53) lo hizo en el último mes; mientras que solo el 7.1\% (23) refiero haber consumido alcohol en la última semana.

La Secretaria de Salud (2019) reporta que el consumo de drogas representa un considerable problema de salud pública en México, refiere que en la actualidad existe una transición epidemiológica que apunta hacia tres vertientes: la disminución en la edad de inicio de consumo, el incremento del consumo entre adolescentes y una importante incursión del género femenino, sobre todo las jóvenes, en los contextos del uso de drogas. En lo que respecta al consumo de alcohol por género, las diferencias han disminuido en los últimos años, la moderación de la mujer impuesta por los códigos sociales, la exentó durante mucho tiempo de las consecuencias negativas vinculadas al uso y/o abuso de alcohol, pero la tendencia actual al disminuir las diferencias de género, además de transformar los roles, también cambio las actitudes ante el consumo de alcohol (Ahumada et al., 2017).

Míguez \& Permuy (2017), documentaron el caso de una población adolescente (14-18 años) de España, donde la prevalencia de consumo de alcohol ya es superior en las mujeres (82.9\%) en relación a los hombres (80.9\%). En las últimas décadas el interés por el consumo de alcohol en mujeres ha evidenciado la necesidad de ampliar el conocimiento sobre las particularidades de esta población y ha favorecido un incremento en las investigaciones sobre las diferencias de género.

Actualmente diversos estudios aportan datos respecto al consumo de alcohol en relación al género, prueba de ello Giménez et al., (2018), encontraron en 894 jóvenes españoles que el $99.4 \%$ habían consumido alcohol, sin mostrar diferencias significativamente estadísticas entre el 99\% de hombres y el $99.7 \%$ de mujeres que lo han hecho. Mientras que el $89.7 \%$ de hombres y el $88.5 \%$ de mujeres refirieron haber experimentado episodios de embriagues. Así mismo, Alonso et al., (2018) en estudiantes mexicanos encontraron que existen diferencias significativas para el tipo de consumo por sexo $\left(\chi^{2}=11.07, p<.001\right)$, se observó que el consumo de bajo riesgo (30.3\%) y el de riesgo es mayor en el sexo femenino (33.3\%); sin embargo, con respecto al consumo perjudicial, es superior en el sexo masculino (59.1\%). De igual forma, Frayre et al., (2018), evidenciaron en un estudio en 276 adolescentes mujeres del estado de Campeche que el $57.6 \%$ declaro haber consumido alcohol alguna vez en la vida, iniciando aproximadamente su consumo a los 14.3 años.

Durante el inicio del consumo de alcohol se encuentran un sinnúmero de factores sociales y personales que influyen en esta conducta, como son las creencias. En la cultura universitaria se cuentan versiones 
positivas sobre los efectos del consumo de alcohol que actúan como reguladoras de esta conducta de consumo. Estas creencias se encuentran estrechamente relacionadas con los beneficios y barreras hacia el consumo, pues si las creencias de las universitarias hacia los efectos del consumo son de beneficio y ocasiona sentimientos agradables, esto irremediablemente incrementará la probabilidad de que las jóvenes se expongan más fácilmente a situaciones de uso y abuso de la sustancia (Oliva, 2014).

La formación de una creencia nace desde el interior de una persona, esta surge como consecuencia de las propias doctrinas y los valores morales, aunque también está influída por factores externos y el entorno social. Al respecto, Castaño et al., (2013) documentaron en 538 estudiantes universitarios colombianos, que las expectativas, creencias, la publicidad, las costumbres familiares y sociales, tienen una relación favorable para el consumo de alcohol.

Asímismo, Londoño \& Carrasco (2019) realizaron un estudio comparativo entre jóvenes colombianos y chilenos con la finalidad de conocer las diferencias en las creencias relacionadas con el consumo de alcohol en 1,602 participantes. Los resultados indicaron que en ambas poblaciones la media más baja fue la de los beneficios del consumo de alcohol, la mayoría de los jóvenes chilenos presento un nivel de creencias de riesgo moderado o alto en los cuatro factores evaluados, al igual que los participantes colombianos. Asímismo, los colombianos, en comparación con los chilenos, presentaron de manera significativa claves disparadoras de consumo más altas, mayor percepción de riesgo y vulnerabilidad, mayor percepción de beneficios percibidos del consumo y menor percepción de barreras para dejar de consumir.

En México, existe evidencia de diversos estudios sobre las creencias y el consumo de alcohol; en Monterrey, Nuevo León, Alonso et al., (2014) documentaron los pros y contras percibidos por 567 estudiantes de preparatoria, de los que el $45 \%$ estuvo en desacuerdo en que beber es emocionante, una gran mayoría estuvo en desacuerdo en que beber da más valor a las personas, sin embargo el $20.3 \%$ de los estudiantes estuvieron de acuerdo en que beber ayuda a pasarlo bien con los amigos y el $22.8 \%$ señaló que los eventos o fiestas son más divertidas cuando hay alcohol.
Con respecto a las barreras el $56.4 \%$ estuvo muy de acuerdo en que cuando se bebe se puede terminar lastimando a alguien y que el beber puede meter en problemas a las personas con la autoridad (55\%). Los pros o beneficios de consumo de alcohol se relacionaron significativamente con el consumo de alcohol $\left(r_{s}=.280\right.$, $p<.001$ ) lo que significó que, a mayores pros o beneficios de consumo de alcohol, mayor es el consumo de alcohol en los jóvenes.

Específicamente en el estado de Tamaulipas, De los Reyes et al., (2014), encontraron en 233 estudiantes de preparatoria de igual manera una relación positiva y significativa entre los pros o beneficios percibidos de consumo de alcohol con el consumo de alcohol $\left(r_{s}=.204\right.$, $p<.002)$, además documentaron una relación negativa y significativa de los contras con el consumo de alcohol $\left(r_{s}=\right.$ -.142, $p<.030$ ), lo cual significa que a menores contras de consumo de alcohol mayor será el consumo de alcohol. Del mismo modo Telumbre \& Sánchez (2014) demostraron en 335 adolescentes de Guerrero que predominaron los beneficios sobre las barreras de consumo de alcohol, algunos de los beneficios que fueron mayoría se relacionaban con la percepción de los adolescentes de que beber alcohol da más valor, da seguridad, hace las fiestas y celebraciones más divertidas y te hace sentir más relajado y menos tenso.

En síntesis, los estudios previos en el contexto del alcohol en entornos universitarios ponen de manifiesto una tendencia al alza del consumo por parte de las mujeres. Igualmente, estudios nacionales e internacionales señalan la relación entre las creencias y el consumo de alcohol. Según lo expuesto, el propósito del presente estudio fue determinar la prevalencia del consumo y las creencias sobre el consumo de alcohol estudiando la relación entre creencias y consumo de alcohol en mujeres universitarias.

\section{MÉTODO}

\section{Diseño y Participantes}

Estudio de enfoque cuantitativo de tipo descriptivo, transversal, de carácter correlacional, la población estuvo constituida por estudiantes del género femenino, de una institución privada de nivel superior de la cuidad de $\mathrm{H}$. Matamoros Tamaulipas, México, que aceptaron 
participar y que se encontraban en el momento de la recolección de datos. La muestra estuvo conformada por 138 estudiantes universitarias.

\section{Instrumentos}

Se utilizó un cuestionario sociodemográfico y de prevalencia de consumo de alcohol (CDPPCA) formado por dos secciones, la primera contiene cinco ítems sociodemográficos. En la segunda sección se indaga sobre la prevalencia de consumo de alcohol alguna vez en la vida, en el último año, en el último mes y en la última semana, con respuesta dicotómica y tres ítems de respuesta abierta.

Para medir las creencias sobre el consumo de alcohol, se utilizó la Escala de Balance Decisional para el Consumo del Alcohol que fue elaborada por Maddock (1997). Está integrada por quince ítems, ocho miden pros y siete miden contras del consumo de alcohol. Consta de una escala de respuesta tipo likert donde 1 equivale a muy en desacuerdo, 2 en desacuerdo, 3 de acuerdo y 4 muy de acuerdo. En relación con los pros, el rango va de 8 a 32, por lo que, a mayor puntuación, mayor percepción de pros hacia el consumo de alcohol. En cuanto a la escala de contras el rango va de 7 a 28 , por lo que, a mayor puntuación, mayor es la percepción de contras hacia el consumo de alcohol. En el presente estudio se reportó una consistencia interna de Alpha de Cronbach de .81 .

\section{Procedimiento}

Para la recolección de los datos, inicialmente se contó con la aprobación por el Comité de Investigación y Ética de la Unidad Académica Multidisciplinaria Matamoros UAT, se solicitó la autorización de los directivos de la institución educativa para la recolección de datos, este estudio se apegó a lo dispuesto en el Reglamento de la Ley General de Salud en Materia de investigación para la salud (secretaria de salud (SSA), 1987), así también con el consentimiento informado de las universitarias. Los instrumentos fueron autoaplicables y anónimos.

\section{Análisis de datos}

Los datos fueron analizados por medio del programa estadístico SPSS versión 23 para Windows, se utilizó estadística descriptiva e inferencial para las variables del estudio, para dar respuesta a los objetivos planteados se aplicó el coeficiente de correlación de Pearson, teniendo en cuenta un intervalo de confianza del $95 \%$, el nivel de significancia se fijó en $5 \%$ para todos los análisis.

\section{RESULTADOS}

La edad de las participantes en el estudio osciló entre 18 y 35 años, con una media de 21.3 años ( $D E=2.6$ ). El $82.6 \%$ se encontraban solteras y el $64.5 \%$ estudiaban y trabajaban en el momento de la entrevista. El mayor porcentaje (38\%) de las encuestadas estudiaban la carrera de Comercio Internacional y el $47.1 \%$ del total de la muestra cursaban el séptimo cuatrimestre.

Para dar cumplimiento al primer objetivo en lo que se refiere al consumo de alcohol, en la Tabla 1 se aprecia la prevalencia de consumo de alcohol alguna vez en la vida, el 92.8\% (IC 95\% .88-.97) de las participantes refirieron haber consumido alcohol en algún momento de su vida, la edad de inicio de consumo de alcohol en las participantes presento una media de 16.7 años ( $D E=1.7$ ), de igual forma, el $77.5 \%$ (IC 95\% .70-.85) refirió haber consumido alcohol en el último año, mientras que el $55.1 \%$ (IC 95\% .47-.63) en el último mes y en la última semana el $29 \%$ (IC 95\% .21-.37) restante. El $61.6 \%$ de las participantes consumen mayormente diversidad de bebidas.

Tabla 1. Prevalencia de consumo de alcohol

\begin{tabular}{|c|c|c|c|c|c|c|}
\hline & \multicolumn{2}{|c|}{$\mathrm{Si}$} & \multicolumn{2}{|c|}{ No } & \multicolumn{2}{|c|}{ IC al 95\% } \\
\hline & $f$ & $\%$ & $f$ & $\%$ & $\mathrm{LI}$ & LS \\
\hline Alguna vez en la vida & 128 & 92.8 & 10 & 7.2 & .88 & .96 \\
\hline En el último año & 107 & 77.5 & 31 & 22.5 & .71 & .84 \\
\hline En el último mes & 76 & 55.1 & 62 & 44.9 & .47 & .63 \\
\hline En la última semana & 40 & 29 & 98 & 71 & .22 & .36 \\
\hline
\end{tabular}

En lo que corresponde al objetivo 2, con respecto a las creencias (Tabla 2), los pros (beneficios) de consumo de alcohol que las estudiantes refirieron, sobresalen los siguientes: el 51.4\% está de acuerdo que beber alcohol te 
hace sentir más relajado y menos tenso con la gente, mientras que el $49.3 \%$ están muy de acuerdo que el beber hace que las personas se sientan más contentas y el $35.5 \%$ refirió que el beber es emocionante. Contrariamente el $40.6 \%$ refirió estar muy en desacuerdo con que beber dé más seguridad en uno mismo.

Tabla 2. Beneficios percibidos (pros) del consumo de alcohol

\begin{tabular}{|c|c|c|c|c|c|c|c|c|}
\hline & \multicolumn{2}{|c|}{$M D$} & \multicolumn{2}{|c|}{$D$} & \multicolumn{2}{|c|}{ DA } & \multicolumn{2}{|c|}{ MA } \\
\hline & $f$ & $\%$ & $f$ & $\%$ & $f$ & $\%$ & $f$ & $\%$ \\
\hline $\begin{array}{l}\text { Beber es } \\
\text { emocionante }\end{array}$ & 29 & 21 & 57 & 41.3 & 49 & 35.5 & 3 & 2.2 \\
\hline $\begin{array}{l}\text { Beber da más } \\
\text { valor a las } \\
\text { personas }\end{array}$ & 48 & 34.8 & 28 & 20.3 & 50 & 36.2 & 12 & 8.7 \\
\hline $\begin{array}{l}\text { Cuando se } \\
\text { bebe hace que } \\
\text { las personas se } \\
\text { sientan más } \\
\text { contentas }\end{array}$ & 24 & 17.4 & 37 & 26.8 & 68 & 49.3 & 9 & 6.5 \\
\hline $\begin{array}{l}\text { Después de } \\
\text { unas cuantas } \\
\text { copas se puede } \\
\text { hablar mejor }\end{array}$ & 44 & 31.9 & 54 & 39.1 & 38 & 27.5 & 2 & 1.4 \\
\hline $\begin{array}{l}\text { Beber me hace } \\
\text { sentir más } \\
\text { relajado y } \\
\text { menos tenso a } \\
\text { la gente }\end{array}$ & 28 & 20.3 & 25 & 18.1 & 71 & 51.4 & 14 & 10.1 \\
\hline $\begin{array}{l}\text { Beber ayuda a } \\
\text { pasarla bien } \\
\text { con los amigos }\end{array}$ & 29 & 21 & 54 & 39.1 & 45 & 32.6 & 10 & 7.2 \\
\hline $\begin{array}{l}\text { Los eventos } \\
\text { (fiestas) son } \\
\text { más divertidos } \\
\text { cuando hay } \\
\text { alcohol }\end{array}$ & 39 & 28.3 & 61 & 44.2 & 32 & 23.2 & 6 & 4.3 \\
\hline $\begin{array}{l}\text { Beber da más } \\
\text { seguridad de sí } \\
\text { mismo }\end{array}$ & 56 & 40.6 & 52 & 37.7 & 23 & 16.7 & 7 & 5.1 \\
\hline
\end{tabular}

Nota: $\mathrm{f}=$ Frecuencia; $\mathrm{MD}=$ Muy en Desacuerdo; $\mathrm{D}=$ En Desacuerdo; $\mathrm{DA}=\mathrm{De}$ Acuerdo; MA=Muy de Acuerdo.

Con respecto a los contras (barreras) de consumo de alcohol percibidos por las estudiantes universitarias, un $53.6 \%$ de las participantes refieren que se puede lastimar a alguien accidentalmente por la forma de beber, además el $50 \%$ describieron que beber puede meter en problemas a las personas con la autoridad, que cuando se bebe mucho se puede lastimar a personas allegadas y además que cuando se bebe se puede terminar lastimando a alguien. En general, la mayoría de las participantes presentaron índices más altos sobre las barreras para el consumo de alcohol.

Para responder al objetivo tres que propone describir la relación entre los pros y contras del consumo de alcohol alguna vez en la vida y en el último año, se aplicó el coeficiente de correlación de Pearson. Las prevalencias de consumo de alcohol mostraron una relación positiva y significativa, con el índice de pros para el consumo de alcohol con los diferentes tipos de consumo (Tabla 3), encontrando una correlación positiva entre motivos favorables y consumo.

Tabla 3. Relación entre pros y prevalencia de consumo de alcohol

\begin{tabular}{lc}
\hline & $r$ \\
\hline Consumo alguna vez en la vida & $.330^{* *}$ \\
Consumo en el último año & $.370^{* *}$ \\
Consumo en el último mes & $.278^{* *}$ \\
Consumo en la última semana & $.342^{* *}$ \\
${ }^{* *} p<.01$ &
\end{tabular}

\section{DISCUSIÓN}

El presente estudio permitió determinar las creencias relacionadas con el consumo de alcohol y el tipo de consumo de alcohol en 138 mujeres universitarias de una universidad privada de la $\mathrm{Cd}$. de $\mathrm{H}$. Matamoros Tamaulipas, México. En relación al consumo de alcohol los resultados difieren de lo reportado en México por la ENCODAT (2017) donde el 62.6\% de las mujeres encuestadas refirieron un consumo de alguna vez en la vida, así mismo el 39\% de ellas tuvieron contacto con el alcohol en el último año. Esta diferencia en los hallazgos encontrados puede deberse a la condición laboral y marital en la que se encontraban las participantes en el momento de la entrevista, dado que un alto porcentaje de ellas son laboralmente activas (64.5\%), y la mayoría de ellas refirieron estar solteras (82.6\%), el hecho de vivir en pareja podría significar una mayor y los requerimientos familiares que exijan pasar más tiempo en el hogar, entre otros. Además, el hecho de que podría entenderse que los solteros tienen mayor disponibilidad de dinero para gastar individualmente que los casados.

La mitad de las participantes refieren estar de acuerdo que beber hace sentir más relajada y con menos tensión hacia las personas, reportando sentirse más contentas cuando beben, contrariamente poco menos de la mitad está en desacuerdo en que las reuniones son más divertidas cuando hay alcohol, además que el beber es emocionante y les da más valor y seguridad. Dichos resultados coinciden con lo encontrado por De Los Reyes et al., (2014), donde reportaron que la mayoría de sus 
encuestados manifestaron estar en desacuerdo con el hecho de que consumir alcohol les genere beneficios hacia su persona. Esta similitud en los resultados probablemente pudiera deberse a que las participantes de ambos estudios comparten características semejantes como el género y rango de edad. Con respecto a los contras o barreras de consumo de alcohol, la mitad de las participantes refieren que se puede lastimar a alguien accidentalmente por la forma de beber, además describieron que tomar puede meter en problemas a las personas con la autoridad, estos resultados difieren con lo reportado por Londoño \& Carrasco en el 2019, quienes al realizar un estudio en una muestra de jóvenes colombianos encontraron una menor percepción de barreras para dejar de consumir alcohol.

Los resultados contradictorios con respecto a los pros y contras de los participantes podrían justificase de la siguiente manera, por una parte, el estar conscientes de las consecuencias y daños que genera el consumir alcohol, lo cual pudiera contribuir a no consumirlo y por otra parte conociendo las consecuencias del uso y abuso de esta substancia, realizar la práctica de consumirlo como la posibilidad de oportunidad para poder socializar con pares. Al respecto Albarracín \& Muñoz (2008) documentan que el consumo alcohol hace parte de la elección y socialización entre amigos, dado que debe existir un consentimiento por parte de los otros, lo cual impide ser descartado socialmente por parte de quienes sí consumen alcohol.

Entre las creencias y el consumo de alcohol, los resultados mostraron relación positiva y significativa con el índice de pros (beneficios) para el consumo de alcohol con todos los tipos de consumo, en la línea de estudios como el de Alonso et al. (2014) en jóvenes regiomontanos. Esto pudiera indicarnos que la percepción de beneficios ligados al consumo de alcohol puede reducir la percepción de riesgo y aumentar la probabilidad de repetición de la conducta (González et al., 2014). Con los hallazgos discutidos anteriormente se confirma que existe una correlación positiva entre las creencias favorables hacia el consumo y la conducta de beber en las mujeres universitarias de la muestra.

\section{Conclusiones}

La prevalencia de consumo de alcohol se sigue manteniendo en niveles elevados en los entornos universitarios. Las creencias favorables hacia el consumo se relacionan positivamente con la conducta de beber entre las universitarias. Se hace por tanto necesario continuar profundizando con estudios de investigación para detectar dónde se generan los comportamientos nocivos en las mujeres universitarias y con base en los resultados, implementar programas de prevención e intervención para evitar, reducir o retardar el uso y abuso del consumo de alcohol.

\section{Conflicto de interés}

Los autores no tienen conflictos de intereses que declarar.

\section{REFERENCIAS}

Ahumada, J., Gámez, M., \& Valdez, C. (2017). El Consumo de alcohol como problema de salud pública. Ra Ximhai, 13(2), 13-24.

Albarracín, M. O., \& Muñoz, O. L. (2008). Factores asociados al consumo de alcohol en estudiantes de los dos primeros años de carrera universitaria. Liberabit, Revista de psicología, 14, 49-61.

Alonso, M. M., López K., Rodríguez, L., Esparza S., Alonso, B. A., \& Alonso, M. T., (2014). Pros, contras, resiliencia y motivaciones para el consumo de alcohol y tabaco en jóvenes de preparatoria. Un estudio multiregional: el caso de Monterrey Nuevo León. En: López K. S., Alonso M. M., Armendáriz N. A., Guzmán F. R., Alonso B. A. \& Rodríguez L. (2014). Experiencias de investigación de la red mexicana de facultades y escuelas de enfermería: Predicción del consumo de alcohol y tabaco en jóvenes de preparatoria (pp. 302 316), Monterrey, Nuevo León, México. Universidad Autónoma de Nuevo León.

Alonso, M. M., Un, L., Armendáriz, N. A., Navarro, E., \& López, M. A., (2018). Sentido de coherencia y consumo de alcohol en jóvenes universitarios. Investigación y Ciencia, 26(75).

Castaño, G.A., García, J. A., \& Marzo J. C., (2014). Consumo de alcohol y factores intervinientes en estudiantes universitarios. Revista Cubana de Salud Pública, 40(1), Pp. 47-54.

De los Reyes L. R., Moreno V., Vázquez, M. G., Vásquez, M. G. E., Ramírez S. A., \& Ramos S. (2014). Pros, contras, resiliencia y motivaciones para el consumo de alcohol y tabaco en jóvenes de preparatoria. Un estudio multiregional: el caso de Cd. Victoria, Tamaulipas. En: López K. S., Alonso M. M., Armendáriz N. A., Guzmán F. R., Alonso B. A. \& Rodríguez L. (2014). Experiencias de investigación de la red mexicana de facultades y escuelas de enfermería: Predicción del consumo de alcohol y tabaco en jóvenes de preparatoria (pp. 451475), Monterrey, Nuevo León, México. Universidad Autónoma de Nuevo León.

Encuesta Nacional de Consumo de Drogas Alcohol y Tabaco 2016-2017 (ENCODAT, 2017) Consumo de alcohol: prevalencias Globales, patrones de consumo y variaciones estatales. Recuperado de: 
https://www.gob.mx/cms/uploads/attachment/file/246052/hojasre sumen_Alcohol-V3.pdf

Frayre, A. D., Jordán, M. L., López M. A., \& Telumbre, J. Y. (2018). Autoeficacia y Consumo de Alcohol en Mujeres Adolescentes. Nure Investigación, 95, 1-9.

García, P., Hinojosa, L., Salazar, M. E., Jiménez, A. A., Guerra, J. A., \& Olivares, O. A. (2016). Perfil sociodemográfico y consumo de alcohol en adolescentes de bachillerato. Paraninfo Digital Monográfico De Investigación En Salud, X(25).

Giménez, C., Ruiz, E., Gil, B., García, J. E., \& Ballester, R.L, (2018). Alcohol y juventud ¿existen diferencias en consumo de hombres y mujeres según edad de inicio? International Journal of Developmental and Educational Psychology, 2(1). doi:10.17060/ijodaep.2018.n1.v2.123

González, B., Gómez, J. A., Gras M. E., \& Planes M., (2014). Búsqueda de sensaciones y consumo de alcohol: el papel mediador de la percepción de riesgos y beneficios. Anales de Psicología, 30(3), 1061-1068. doi:10.6018/analesps.30.3.170831

Londoño, C., \& Carrasco, S. F. (2019). Creencias sobre el consumo de alcohol en jóvenes colombianos y chilenos. Acta Colombiana de Psicología, 22(2), 178-185. doi:10.14718/ACP.2019.22.2.9

Maddock, J.E. (1997). Developmente and validation of decisional balance and process of change inventories for heavy episodic drinking. Tesis de maestría no publicada, University of Rhode Island, E.E. U.U.

Míguez, M. C., \& Permuy B., (2017). Características del alcoholismo en mujeres. Revista de la Facultad de Medicina. Universidad Nacional de Colombia, 65(1), 15-22. doi:10.15446/revfacmed.v65n1.57482

Oliva-Moreno, J. L., (2014). Pros y contras percibidos del consumo de alcoholy consumo de alcohol en estudiantes de preparatoria. (Tesis de Maestría no publicada). Universidad Autónoma de Nuevo León, México.

Organización Mundial de la Salud (2018). Nota informativa emitida el 21 de septiembre de 2018. Recuperado de: http://www.who.int/es/news-room/fact-sheets/detail/alcohol

Organización Mundial de la Salud (2014). Glosario de términos de alcohol y drogas. Ministerio de sanidad y consumo, Madrid, España. Disponible en: https://www.who.int/substance_abuse/terminology/lexicon_alcoho I drugs spanish.pdf

Organización Panamericana de la Salud (2015). Informe de situación regional sobre el alcohol y la salud en las Américas. Recuperado de: https://www.paho.org/hq/dmdocuments/2015/alcohol-Informesalud-americas-2015.pdf

Prochaska, J. O. \& Di Clemente, C. C. (1997). The transteoretical model and stanges of change. Heal Behavior and Healt Eduaction. (2a.ed.) 6066.

Secretaria de salud (2014). Encuesta Nacional de consumo de drogas en estudiantes. Resultados Tamaulipas Recuperado de: http://omextad.salud.gob.mx/contenidos/vigilancia/Tamaulipas/H R_Tamaulipas.pdf
Secretaria de salud (2019) Informe sobre la Situación del Consumo de Drogas en México y su atención integral. Recuperado de: https://www.gob.mx/cms/uploads/attachment/file/477564/Inform e_sobre_la_situacio_n_de_las_drogas_en_Me_xico_pdf

Secretaria de Salud. (1987). Reglamento de la Ley General de Salud en Materia de Investigación para salud. México. Disponible en: http://www.salud.gob.mx/unidades/cdi/nom/compit/rlgsmis.html

Telumbre J. Y., \& Sánchez B. E., (2015). Percepción de Barreras y Beneficios del Consumo de Alcohol en Adolescentes. Nure Investigación, 76, 1 10. 